\title{
Primary pancreatic paraganglioma: a case report and literature review
}

\author{
Shengrong Lin ${ }^{1}$, Long Peng ${ }^{1}$, Song Huang ${ }^{2}$, Yong Li ${ }^{1}$ and Weidong Xiao ${ }^{1 *}$
}

\begin{abstract}
Backgroud: Primary pancreatic paraganglioma is an extremely rare extra-adrenal paraganglioma.

Case presentation: We report a case of primary pancreatic paraganglioma undergoing middle segment pancreatectomy in a 42-year-old woman. Histological examination showed that the tumor was composed of welldefined nests of cuboidal cells separated by vascular fibrous septa, forming the classic Zellballen pattern. The chief cells showed positive staining to neuron-specific enolase, chromogranin A, synaptophysin, and the chief cells were surrounded by S-100 protein-positive sustentacular cells. The patient has remained tumor free for 12 months after surgery. A brief discussion about the histopathological features, clinical behavior, and treatment of primary pancreatic paraganglioma, and review of the relevant literature is presented.

Conclusions: Primary pancreatic paraganglioma is a rare clinical entity, its diagnosis mainly depends on histopathological and immunohistochemical examinations. Complete surgical resection is the first choice of treatment and close postoperative follow-up is necessnary.
\end{abstract}

Keywords: Pancreas, Paraganglioma, Middle segment pancreatectomy

\section{Background}

Paragangliomas are rare neuroendocrine tumors (NETs) that arise from the extra-adrenal chromaffin cells of the autonomic nervous system, with an average annual incidence rate of only 2 to 8 per 1 million adults. Paragangliomas could derive from the extra-adrenal chromaffin cells of the sympathetic paravertebral ganglia of the thorax, abdomen, and pelvis, also arise from the parasympathetic ganglia located along the glossopharyngeal and vagal nerves in the neck and at the base of the skull. However, primary pancreatic paraganglioma is extremely rare. Herein, we present a case of primary pancreatic paraganglioma and review of the literature.

\section{Case presentation}

A 42-year-old woman presented in September 2014 with recurrent upper abdominal pain for 3 months. She had no history of hypertension, headache, and palpitation. A physical examination revealed slight upper abdominal tenderness. Laboratory test results including liver

\footnotetext{
*Correspondence: frankxwd@126.com

'Department of General Surgery, The First Affiliated Hospital of Nanchang

University, No.17 Yongwai Zhengjie, Nanchang, 330006 Jiangxi, China

Full list of author information is available at the end of the article
}

function, renal function, and blood glucose were within normal ranges. Serum levels of CEA, CA19-9, and CA125 were normal. The level of 24-h urinary norepinephrine excretion was also normal. Unenhanced computed tomography $(\mathrm{CT})$ revealed a $5.2 \mathrm{~cm} \times 6.3 \mathrm{~cm}$, solid, low density tumor on the body of the pancreas. On contrast-enhanced CT, the tumor demonstrated marked enhancement in the arterial phase (Fig. 1). Dilation of the pancreatic duct was noted at the tail of pancreas. No biliary dilation or liver lesions were detected. A diagnosis suspicion of a pancreatic neuroendocrine tumor was made before operation. The patient underwent middle segment pancreatectomy. The proximal pancreas was transected using a linear stapler and continuous suture using 4-0 prolene, the stump of the distal pancreas was anastomosis to the jejunum with duct-tomucosa pancreaticojejunostomy. The two resection margins were frozen section to confirm tumor-free. During the operation, the patient's blood pressure remained stable. Histological examination showed that the tumor was composed of well-defined nests of cuboidal cells separated by vascular fibrous septa, forming the classic Zellballen pattern (Fig. 2a). The chief cells showed positive staining to neuron-specific enolase (NSE) (Fig. 2b), 


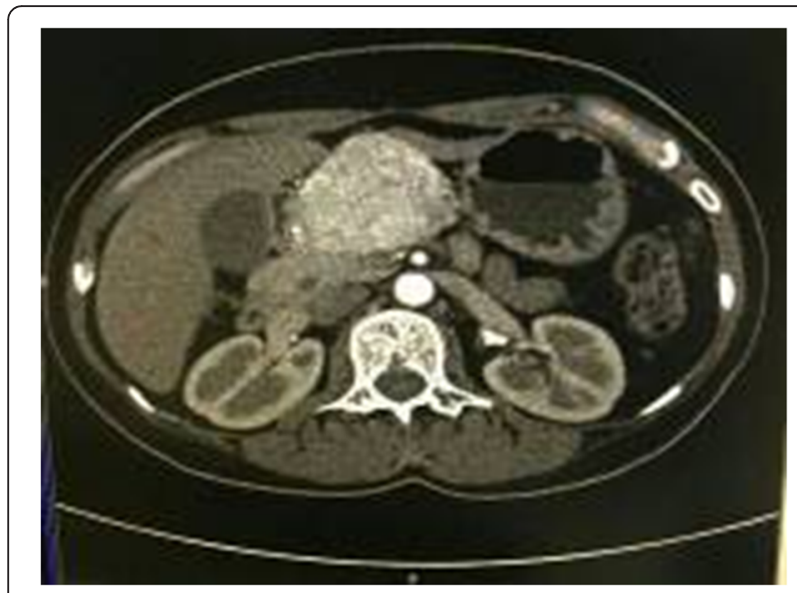

Fig. 1 On contrast-enhanced CT, the tumor demonstrated marked enhancement in the arterial phase

chromogranin A (CgA), synaptophysin (Syn) (Fig. 2c), but showed negative response to vimentin (Vim), endomysial (EMA), cytokeratin (CK), insulin, and glucagon. The chief cells were surrounded by S-100 proteinpositive sustentacular cells (Fig. 2d). The Ki67 labeling index was $1 \%$ where no mitoses were observed. Region lymph nodes and the resected margins were free of tumor cells. Taking the morphological and immunohistochemical features into account, the diagnosis of primary pancreatic paraganglioma was confirmed. The patient's postoperative course was uneventful and discharged on the 8th postoperative day. The patient received no subsequent adjuvant treatment, and had remained tumor free for 12 months after surgery.

\section{Discussion}

Primary paraganglioma that arises in the pancreas is rare. Whether this tumor type is an extension of a retroperitoneal tumor of true visceral origin, derived from ectopic paraganglia, remains unknown. To the best of our knowledge, only 21 cases of pancreatic paraganglioma having been reported from 1943 to the present in the literature (Table 1) [1-18]. The mean age of the 21 cases reported in the literature was 57.6 years ranging from 19 to 85 years. Of those patients, 16 were women and five were men with the ratio of 3.2:1. The tumor was located in the head of the pancreas in 15 patients, 2 in the body, and 4 in the tail. The mean size of the tumors was $6.1 \mathrm{~cm}$. Four cases were considered malignant, and six cases showed functional activity. Herein, we presented a 42-year-old woman with primary pancreatic paraganglioma, which located in the body of the pancreas. The patient had no symptoms of catecholamine excess, and the blood pressure remained stable during the operation, the norepinephrine levels was normal, therefore nonfunctional pancreatic paraganglioma was diagnosed.

The location of pancreatic paragangliomas can usually be identified by abdominal ultrasonography, CT, or magnetic resonance imaging. In our current case, the pancreatic paraganglioma appeared as a solid mass on the body of the pancreas on CT scans, and marked enhancement was found on contrast-enhanced CT. The preoperative diagnosis of pancreatic paraganglioma is difficult, especially in nonfunctional cases. Functional cases are easier to diagnose because having symptoms of catecholamine excess such as hypertension, headache, and palpitation, and the urinary catecholamines are elevated. The confirmed diagnosis of

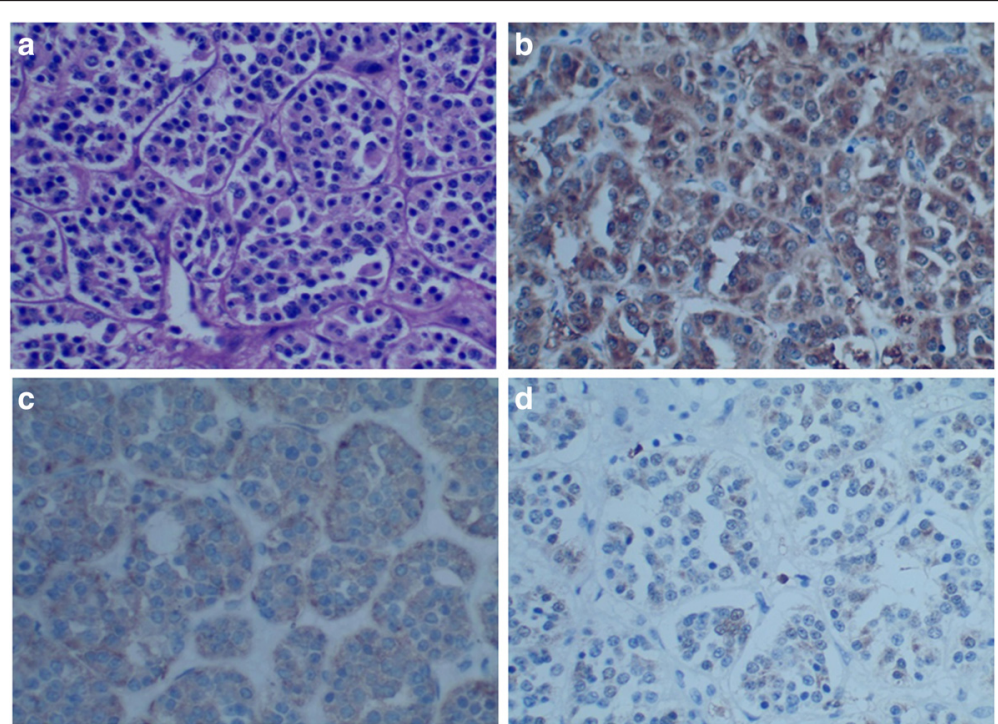

Fig. 2 Pathology slides of the resected specimen with different stains. a Hematoxylin and eosin staining demonstrating the classic Zellballen pattern of paraganglioma $(H E, \times 400)$. b The chief cells showed positive staining to NSE $(I H C, \times 400)$. c The chief cells showed positive staining to Syn $(I H C, \times 400)$. d The sustentacular cells showed positive staining to $S-100(I H C, \times 400)$ 
Table 1 Twenty-one cases of pancreatic paraganglioma in the literature

\begin{tabular}{|c|c|c|c|c|c|c|c|c|c|c|}
\hline Author & Year & Age (y) & Sex & Location & Size $(\mathrm{cm})$ & Cystic or Solid & Function & Malignant & Treatment & Survival \\
\hline Goodo [1] & 1943 & 62 & M & Body & 1.5 & Solid & No & No & - & Autopsy \\
\hline Bartley [2] & 1966 & 75 & $\mathrm{~F}$ & Tail & Goose egg & Cystic & Yes & No & DP & NM \\
\hline Bartley [2] & 1966 & 70 & $\mathrm{~F}$ & Head & Walnut & Cystic & Yes & No & TR & NM \\
\hline Cope [3] & 1974 & 72 & $\mathrm{~F}$ & Head & 13 & Cystic & No & No & TR & $2 Y(A)$ \\
\hline Zamir [4] & 1984 & 47 & M & Body & 10 & Cystic & No & No & TR & $6 Y(A)$ \\
\hline Fujino [5] & 1998 & 61 & M & Head & 2.5 & Solid & No & No & PD & $5 Y(A)$ \\
\hline Parithivel [6] & 2000 & 85 & M & Head & 6 & Cystic & No & No & $\mathrm{TR}$ & $3 Y(A)$ \\
\hline Ohkawara [7] & 2005 & 72 & $\mathrm{~F}$ & Head & 4 & Cystic & No & No & TR & NM \\
\hline Perrot [8] & 2007 & 41 & $\mathrm{~F}$ & Tail & 4.2 & Solid & Yes & No & TR & $18 M(A)$ \\
\hline Tsukada [9] & 2008 & 57 & $\mathrm{~F}$ & Head & 2 & Solid & No & No & TR & $4 Y(A)$ \\
\hline Kim [10] & 2008 & 57 & $\mathrm{~F}$ & Head & 6.5 & Solid & No & No & PPPD & NM \\
\hline Paik [11] & 2009 & 70 & $\mathrm{~F}$ & Tail & 4.2 & Solid & No & Yes & DP & NM \\
\hline $\mathrm{He}[12]$ & 2011 & 40 & $\mathrm{~F}$ & Head & 4.5 & Solid & No & No & NM & NM \\
\hline Higa [13] & 2012 & 65 & $\mathrm{~F}$ & Head & 2 & Solid & No & Yes & PD & $10 M(A)$ \\
\hline Al-Jiffry [14] & 2013 & 19 & $\mathrm{~F}$ & Head & 9.5 & Solid & Yes & Yes & PD & $3 Y(A)$ \\
\hline Zhang [15] & 2014 & 50 & $\mathrm{~F}$ & Head & 6 & Solid & Yes & Yes & Laparotomy & $4 Y(D)$ \\
\hline Zhang [15] & 2014 & 63 & M & Head & 4 & Solid & Yes & No & $\mathrm{TR}$ & $3 M(A)$ \\
\hline Borgohain [16] & 2014 & 55 & $\mathrm{~F}$ & Tail & 19 & Solid & No & No & TR & $10 \mathrm{M}(\mathrm{A})$ \\
\hline Straka [17] & 2014 & 53 & $\mathrm{~F}$ & Head & 8.5 & Solid & No & No & PPPD & $49 M(A)$ \\
\hline Meng [18] & 2015 & 54 & $\mathrm{~F}$ & Head & 3 & Solid & No & No & TR & NM \\
\hline Meng [18] & 2015 & 41 & $\mathrm{~F}$ & Head & 6 & Solid & No & No & TR & NM \\
\hline
\end{tabular}

$F$ female, $M$ male, NM not mentioned, $D P$ diatal pancreatectomy, $P D$ pancreaticoduodenectomy, $T R$ tumor resection, $P P P D$ pylorus preserving pancreaticoduodenectomy, $Y$ years, $M$ months, $A$ alive, $D$ dead

paraganglioma mainly depends on histopathological and immunohistochemical findings as following (i) the classic Zellballen pattern composed of chief cells and sustentacular cells within the tumor; (ii) the chief cells showed positive staining to NSE, CgA, Syn, and negative for CK; and (iii) the sustentacular cells showed positive staining to S-100 or GFAP. Pancreatic paragangliomas are potential to be malignant [13-15], but factors predictive of malignant behaviors have not been well characterized. In general, malignant paragangliomas are defined as those that metastasize, recur, or show evidence of local invasion.

The first choice of treatment for primary pancreatic paraganglioma is complete surgical resection. Postoperative $\mathrm{I}^{131}$ metaiodobenzylaguanidine ( $\left.\mathrm{I}^{131}-\mathrm{MIBG}\right)$ radiotherapy has been advocated in cases proven to be malignant [14], chemotherapy and novel biologically targeted drugs could be the other reasonable choice. Although pancreaticoduodenectomy or pylorus preserving pancreaticoduodenectomy is recommended for paraganglioma of the pancreatic head, and distal pancreatectomy for tumors of the pancreatic body or tail, simple tumor enucleation also showed an equally good outcome. In the present case, middle segment pancreatectomy was performed being the tumor located in the body of the pancreas. Middle segment pancreatectomy procedure has the advantage of preserving normal pancreatic parenchyma to the most extent and consequently long-term endocrine and exocrine pancreatic function. The patient had an uneventful postoperative course. Being have no evidence of tumor invasion and metastases, the patient received no subsequent adjuvant treatment, and has been tumor free for 12 months after surgery. The long-term outcome is still in follow-up.

\section{Conclusions}

We report a case of primary nonfunctional paraganglioma on the body of the pancreas. Middle segment pancreatectomy could be a reasonable procedure for such tumor. Pancreatic paraganglioma has malignant potential, and requiring close postoperative follow-up.

\section{Consent}

Written informed consent was obtained from the patient for publication of this Case Report and any accompanying images. A copy of the written consent form is available for review by the Editor-in-Chief of this journal.

\section{Abbreviations}

CgA: chromogranin A; CK: cytokeratin; CT: computed tomography;

EMA: endomysial; MSP: middle segment pancreatectomy;

NETs: neuroendocrine tumors; NSE: neuron-specific enolase;

Syn: synaptophysin; Vim: vimentin. 


\section{Competing interests}

The authors declare that they have no competing interests.

\section{Authors' contributions}

XWD designed the study; LSR analyzed the data and drafted the manuscript; PL collected the data and presented the clinical features; LY, XWD, and LSR performed the operation; HS made the pathologic diagnosis. All authors have read and approved the final manuscript.

\section{Author details}

'Department of General Surgery, The First Affiliated Hospital of Nanchang University, No.17 Yongwai Zhengjie, Nanchang, 330006 Jiangxi, China. 2Department of Pathology, The First Affiliated Hospital of Nanchang University, No.17 Yongwai Zhengjie, Nanchang, 330006 Jiangxi, China.

Received: 24 September 2015 Accepted: 11 January 2016

Published online: 22 January 2016

\section{References}

1. Goodof II, Lischer CE, Louis S. Tumor of the carotid body and the pancreas. Arch Pathol. 1943;35:906-11.

2. Bartley O, Ekdahl PH, Hultén L. Paraganglioma simulating pancreatic cyst. Acta Chir Scand. 1966;132:289-97.

3. Cope C, Greenberg SH, Vidal JJ, Cohen EA. Nonfunctioning nonchromaffin paraganglioma of the pancreas. Arch Surg. 1974;109:440-42.

4. Zamir O, Amir G, Lernau O, Ne'eman Z, Nissan S. Nonfunctional paraganglioma of the pancreas. Am J Gastroenterol. 1984;79:761-63.

5. Fujino Y, Nagata Y, Ogino K, Watahiki H, Ogawa H, Saitoh Y. Nonfunctional paraganglioma of the pancreas: report of a case. Surg Today. 1998;28:209-12.

6. Parithivel VS, Niazi M, Malhotra AK, Swaminathan K, Kaul A, Shah AK. Paraganglioma of the pancreas: literature review and case report. Dig Dis Sci. 2000;45:438-41.

7. Ohkawara T, Naruse H, Takeda H, Asaka M. Primary paraganglioma of the head of pancreas: contribution of combinatorial image analyses to the diagnosis of disease. Intern Med. 2005:44:1195-96.

8. Perrot G, Pavic M, Milou F, Crozes C, Faucompret S, Vincent E. Difficult diagnosis of a pancreatic paraganglioma. Rev Med Interne. 2007;28:701-4

9. Tsukada A, Ishizaki Y, Nobukawa B, Kawasaki S. Paraganglioma of the pancreas: a case report and review of the literature. Pancreas. 2008:36:214-16.

10. Kim SY, Byun JH, Choi G, Yu E, Choi EK, Park SH, et al. A case of primary paraganglioma that arose in the pancreas: the color Doppler ultrasonography and dynamic CT features. Korean J Radiol. 2008;9(Suppl): S18-21.

11. Paik KY. Paraganglioma of the pancreas metastasized to the adrenal gland: a case report. Korean J Gastroenterol. 2009;54:409-12.

12. He J, Zhao F, Li H, Zhou K, Zhu B. Pancreatic paraganglioma: a case report of CT manifestations and literature review. Quant Imaging Med Surg. 2001;1:41-3.

13. Higa B, Kapur U. Malignant paraganglioma of the pancreas. Pathology. 2012; 44:53-5.

14. Al-Jiffry BO, AlNemary $Y$, Khayat $\mathrm{SH}$, Haiba M, Hatem M. Malignant extraadrenal pancreatic paraganglioma: case report and literature review. BMC Cancer. 2013;13:486

15. Zhang $L$, Liao Q, Hu Y, Zhao Y. Paraganglioma of the pancreas: a potentially functional and malignant tumor. World J Surg Oncol. 2014;12:218.

16. Borgohain M, Gogoi G, Das D, Biswas M. Pancreatic paraganglioma: an extremely rare entity and crucial role of immunohistochemistry for diagnosis. Indian J Endocrinol Metab. 2013:17:917-9.

17. Straka M, Soumarova R, Migrova M, Vojtek C. Pancreatic paraganglioma-a rare and dangerous entity. Vascular anatomy and impact on management. J Surg Case Rep. 2014;17:rju074.

18. Meng L, Wang J, Fang SH. Primary pancreatic paraganglioma: a report of two cases and literature review. World J Gastroenterol. 2015;21:1036-9.

\section{Submit your next manuscript to BioMed Central and we will help you at every step:}

- We accept pre-submission inquiries

- Our selector tool helps you to find the most relevant journal

- We provide round the clock customer support

- Convenient online submission

- Thorough peer review

- Inclusion in PubMed and all major indexing services

- Maximum visibility for your research

Submit your manuscript at www.biomedcentral.com/submit 